\title{
Alzheimer's Disease Antibodies Bind Specifically to a Neurofilament Protein in Torpedo Cholinergic Neurons
}

\author{
Joab Chapman, ' Orit Bachar, ${ }^{1}$ Amos D. Korczyn, ${ }^{2}$ Eliyahu Wertman, ${ }^{3}$ and Daniel M. Michaelson ${ }^{1}$ \\ 'Department of Biochemistry, The George S. Wise Faculty of Life Sciences, Tel Aviv University, and 'Department of \\ Neurology, Ichilov Medical Center, Sackler School of Medicine, Tel Aviv University, Ramat Aviv 69978, Israel, and \\ ${ }^{3}$ Department of Neurogeriatrics, Ezrat Nashim Hospital, Jerusalem 91001, Israel
}

\begin{abstract}
Alzheimer's disease (AD) is characterized by neurofibrillary tangles and neuritic plaques and by the degeneration of central cholinergic neurons. Recent studies indicated the presence of antibodies in the sera and cerebrospinal fluid of AD patients which react with neuronal tissue and which recognize cholinergic neurons. In order to identify the cholinergic antigens against which the $A D$ antibodies are directed, we have recently used the purely cholinergic electromotor neurons of the electric fish Torpedo which are chemically homogenous and cross-react antigenically with mammalian cholinergic neurons. This study revealed that immunoglobulins (lgG) from sera of $A D$ patients bind specifically to an antigen in Torpedo electromotor neurons with an apparent molecular weight of $200 \mathrm{kDa}$. In the present report we attempt to characterize this antigen. The similarity in size of this protein to that of the heavy neurofilament subunit (NF-H) and the association of neurofilaments with plaques and tangles prompted us to examine the possibility that it is a neurofilament protein. Our findings show that IgG from sera of AD patients bind to the NF-H protein of Torpedo cholinergic neurons. Comparison of the binding of $A D$ and control IgG to Torpedo cholinergic NF-H revealed that AD IgG bind to this neurofilament protein more readily than do control IgG. In contrast, $A D$ and control IgG bind similarly to NF-H obtained from the chemically heterogenous Torpedo spinal cord and rat brain. These findings suggest that $A D$ sera contain a repertoire of anti-NF-H IgG and that a subpopulation of these antibodies whose levels are significantly elevated in AD binds to epitopes highly enriched in Torpedo cholinergic NF-H. The possible role of these antibodies in the cholinergic dysfunction in $A D$ and their diagnostic potential are discussed.
\end{abstract}

Alzheimer's discase (AD) is characterized by 2 neurohistological hallmarks, neurofibrillary tangles and neuritic plaques. These structures are particularly pronounced in cortical and hippocampal areas and in th: nuclei of the basal forebrain, which

Reccived Aug. 1, 1988: revised Nov. 28, 1988; accepted Dec. 28, 1988.

We thank Dr. I. Ginsburg for the generous gift of purified rat microtubules. This work was supported in part by a grant to D.M.M. from the Easterson Trust and by a Foulkes Foundation Fellowship to J.C.

Correspondence should be addressed to Daniel M. Michaelson at the above address.

Copyright (c) 1989 Society for Neuroscience $0270-6474 / 89 / 082710-08 \$ 02.00 / 0$ provide most of the cholinergic input to the cortex and hippocampus (Coyle et al., 1983). The severity of these neuropathological changes correlates with the cognitive impairment in $\mathrm{AD}$ (Blessed et al., 1968) as well as with a reduction in central cholinergic parameters (Francis et al., 1985). The cholinergic changes are manifested by degeneration and death of neurons in the basal forebrain and by a concomitant reduction in presynaptic cholinergic markers in the cortex and the hippocampus (Sims et al., 1983). The extent of the cholinergic deficit, its occurrence early in the disease, and its correlation with the cognitive deficit in AD (Francis et al., 1985) all indicate a central role for cholinergic degeneration in the pathogenesis of AD.

Several reports indicated the presence of antibodies in AD sera that react with neuronal tissue (Nandy, 1978; Watts et al., 1981; Fillit et al., 1985) and of antibodies in the cerebrospinal fluid of AD patients that specifically recognize cholinergic neurons in rat brain (McRae-Degueurce ct al., 1987). In view of the marked cholinergic degencration in AD, it would be of interest to characterize the antigens against which the AD antibodies are directed as well as their neuronal specificity. Ideally this should be investigated with a preparation of homogenous purely cholinergic human or other mammalian neurons. Because such preparations are not available, we approached this problem by utilizing antigens from the purely cholinergic electromotor neurons of the electric ray, Torpedo, which are chemically homogenous and cross-react antigenically with human and other mammalian cholinergic neurons (Patrick and Lindstrom. 1973; Kushner, 1984). This study showed that immunoglobulins ( $\mathrm{IgG}$ ) from sera of $\mathrm{AD}$ patients bind to a specific $200-\mathrm{kDa}$ antigen in the cell bodies and axons of Torpedo cholinergic neurons and that the levels of such antibodies are significantly elevated in AD patients (Chapman et al., 1988).

In the present report we characterize the $200-\mathrm{kDa}$ Torpedo antigen (PK200) against which the AD IgG are directed. The apparent molecular weight of PK200 is similar to that of the heavy neurofilament subunit (NF-H). This similarity and the association of neurofilaments and cytoskeleton-like elements with plaques and tangles (Anderton et al., 1982; Sternberger et al., 1985; Grundke-Iqbal et al., 1986a; Selkoe and Abraham, 1986; Perry et al., 1987) prompted us to examine the possibility that PK200 is a neurofilament proteiri. Our findings showed that AD sera contain a repertoire of anti-NF-H IgG and that a subpopulation of these antibodies is specific to AD and binds to NF-H epitopes whose levels are significantly higher in neurofilaments of Torpedo cholinergic neurons than in those obtained from heterogenous neuronal preparations. 


\section{Materials and Methods}

\section{Preparation of antigens}

Torpedo cholinergic cell bodies and axons. Torpedo ocellata were caught live off the coast of Tel Aviv and maintained in seawater aquariums for up to 3 months prior to use. Electromotor cholinergic cell bodies were purified from homogenates of freshly excised Torpedo electric lobes by density gradient centrifugation as described by Dowdall et al. (1976). Electromotor nerves were excised and were either used for the preparation of neurofilaments (see below) or extracted by homogenization $(10 \% \mathrm{wt} / \mathrm{vol})$ in $10 \mathrm{~mm}$ phosphate buffer $(\mathrm{pH} 7.4)$ which contained 140 mм $\mathrm{NaCl}$ and $1 \%$ sodium dodecyl sulfate (SDS) (wt/vol).

Purification of neurofilaments. Torpedo neurofilaments were prepared from Torpedo electric lobes $(\sim 1 \mathrm{gm})$, electromotor axons $(\sim 2 \mathrm{gm})$, and spinal cords $(\sim 2 \mathrm{gm})$, whereas mammalian neurofilaments were prcpared from the brain stem $(\sim 1 \mathrm{gm})$ of adult Sprague-Dawley rats. The excised tissues were soaked in a 100 -fold excess of a solution containing EDTA (2.5 Inm), EGTA (2.5 mm), and $\mathrm{Na}_{2} \mathrm{HPO}_{4}$ ( $1 \mathrm{~mm}$ ), pH 7.0, for $2 \mathrm{hr}$ at room temperature as described by Schlaepfer and Freeman (1978). Neurofilaments were purified from the osmotically swollen tissues according to Willard and Simon (1981). The tissues were removed from the solution and homogenized with a loose-fitting pestle of a Donnie homogenizer. $\mathrm{NaCl}(1 \mathrm{M})$ was added to the disrupted tissues to a final concentration of $0.15 \mathrm{M}$, and the homogenate was centrifuged for $30 \mathrm{~min}$ at $12,000 \times \mathrm{g}$. The supernatant was applied to a discontinuous sucrose gradient containing layers $(3.3 \mathrm{ml}$ each) of $2.0,1.5$, and $1.0 \mathrm{M}$ sucrose in a solution of $\mathrm{NaCl}(0.15 \mathrm{M})$, EDTA $(2.5 \mathrm{~mm})$, EGTA $(2.5$ $\mathrm{mM})$, and $\mathrm{Na}_{2} \mathrm{HPO}_{4}(1 \mathrm{mM})$. The gradient was centrifuged in a SW40.1 rotor for $3 \mathrm{hr}$ at $200,000 \times \mathrm{g}$, and the neurofilaments were collected at the 1.0-1.5 $\mathrm{m}$ sucrose interface. Rat microtubules were purified according to Shelanski et al. (1973). Protein was assayed by the method of Lowry et al. (1951) as modified by Markwell et al. (1978) utilizing bovine serum albumin as standard.

\section{Collection of sera}

Patients ( 4 male, 11 female; age $=77 \pm 5$, mean \pm SD) were diagnosed as suffering from AD by research criteria (McKhann et al., 1984; Zenilcov et al., 1984). The AD patients all had experienced an insidious onset of the disease and had exhibited the disease for at least 2 years, and all were severely demented, with mini mental test scores below $60 \%$ of normal. Normal controls ( 3 male, 12 female; age $=75 \pm 5$ ) were nondemented and had no neurological or immunological diseases. Patients with multi-infarct dementia ( 9 male, 3 female; age $=75 \pm 6$ ) and Parkinson's disease with dementia ( 4 male, 4 female; age $=73 \pm 4$ ) and non-demented patients with cerebrovascular disease $(5$ male, 2 female; age $=71 \pm 6$ ) and Parkinson's' disease (4 male, 1 female; age $=70 \pm 2$ ) were diagnosed by standard clinical criteria.

\section{Immunoblot assay}

The antigen preparations ( $1 \mathrm{mg}$ protein $/ \mathrm{ml}$ ) were boiled for $5 \mathrm{~min}$ in $100 \mathrm{~mm}$ Tris buffer, $\mathrm{pH} 6.8$, which contained $1.8 \% \mathrm{SDS}(\mathrm{wt} / \mathrm{vol})$ and $3 \%$ mercaptoethanol ( $\mathrm{vol} / \mathrm{vol}$ ). Polypeptides were separated by electrophoresis on $12 \times 15 \mathrm{~cm} \mathrm{7.5 \%} \mathrm{SDS-polyacrylamide} \mathrm{gels} \mathrm{utilizing} \mathrm{a} \mathrm{Biorad}$ Protean II Slab Cell (50 mA for $2.5 \mathrm{hr}$ ) (Laemmli, 1970). Unless otherwise specified, the amount of protein loaded per lane was as follows: Torpedo clectromotor perikarya $(50 \mu \mathrm{g})$, neurofilaments isolated from the perikarya (PK; $10 \mu \mathrm{g})$ and axons $(2 \mu \mathrm{g})$ of the electromotor neurons and from Torpedo spinal cord $(4 \mu \mathrm{g})$ and rat brain stem $(10 \mu \mathrm{g})$. This insured that comparable levels of the heavy neurofilament protein NF-H were loaded on to the gels in all experiments. The separated polypeptides were transferred electrophoretically to nitrocellulose membranes (Biorad Transblot Cell at $100 \mathrm{~V}$ for $2 \mathrm{hr}$ ) (Towbin et al., 1979), which were then cut into strips. When the effect of dephosphorylation on the immunoassay was examined, the blots were incubated at $25^{\circ} \mathrm{C}$ for $2.5 \mathrm{hr}$ in $100 \mathrm{~mm}$ Tris $\mathrm{HCl}, \mathrm{pH} 8.0$, which contained $1 \mathrm{~mm}$ phenylmethylsulfonylfluoride and 30 units $/ \mathrm{ml}$ calf intestinal alkaline phosphatase (type VIII, Sigma) or in the same solution devoid of phosphatase (Sternberger and Sternberger, 1983). They were then washed $(3 \times)$ in PBS (140 $\mathrm{mM} \mathrm{NaCl}$ in $10 \mathrm{~mm}$ phosphate buffer, $\mathrm{pH} \mathrm{7.4)} \mathrm{and} \mathrm{overlaid} \mathrm{overnight}$ at $4^{\circ} \mathrm{C}$ with $5 \%$ powdered skim milk in PBS. When the effect of dephosphorylation was not examined, the nitrocellulose strips were transferred directly to the skim milk solution. Following the overnight incubation, the nitrocellulose strips were washed $3 \times$ in PBS $+0.05 \%$ TWEEN ( $\mathrm{vol} / \mathrm{vol})$ and reacted for $2 \mathrm{hr}$ at $25^{\circ} \mathrm{C}$ with human sera diluted in PBS + TWEEN which contained $5 \%$ powdered skim milk. They were then washed and the bound $\operatorname{IgG}$ were detected by peroxidase-conjugated anti-human IgG (Sigma, 1:1000 in PBS + TWEEN for $1 \mathrm{hr}$ at $25^{\circ} \mathrm{C}$ ). The nitrocellulose strips were then washed and developed for $20 \mathrm{~min}$

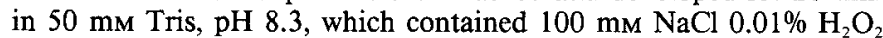
( $\mathrm{vol} / \mathrm{vol}$ ) and $0.6 \mathrm{mg} / \mathrm{ml}$ 4-chloro-1-naphthol. The immunoblots were analyzed by a computerized densitometer (LKB model 2400) and the areas under the peaks recorded. The molecular weights of polypeptides on the SDS-polyacrylamide gels and following the immunoblot assay were determined from the position of marker proteins of known molecular weight. Proteins were visualized with either Coomassie blue (SDS-polyacrylamide gels) or Ponceau S (nitrocellulose strips).

\section{Statistical analysis}

The values for the immunoblot peaks obtained with sera of the AD patients were compared to those of the other groups by means of a Wilcoxon rank order test. Comparison of the proportions of patients in the AD group and in other groups who were positive by a threshold criterion (see Table 1) were done by nonparametric statistical tests (Fisher's exact test or chi-square test) (Colton, 1974). Linear regression analysis was used to calculate the least squares regression line for paired samples, and the probability of a given Pearson $R$ value was evaluated using the $T$ distribution (Colton, 1974).

\section{Results}

The polypeptide composition of the PK and axons of the purely cholinergic Torpedo electromotor neurons and of neurofilaments purified from these preparations is depicted in Figure 1. As can be seen, axonal neurofilaments contain heavy $(H)$, medium $(M)$, and light $(L)$ subunits whose corresponding molecular weights are $200 \mathrm{kDa}, 150 \mathrm{kDa}$, and $63 \mathrm{kDa}$, respectively, as well as a $58-\mathrm{kD}$ a polypeptide. Neurofilaments purified from Torpedo cholinergic PK also contain $H, M$, and $L$ proteins, although the $M$ protein in this preparation is relatively less abundant than that in the axons. Torpedo cholinergic PK neurofilaments have a number of additional polypeptides, of which the $100 \mathrm{kD}$ protein is most prominent (Fig. 1).

The possibility that the Torpedo cholinergic antigen PK200 that is recognized by AD IgG is a neurofilamentous antigen was examined by immunoblot experiments in which the binding of AD IgG to PK200 and to the neurofilament proteins of these neurons was determined and compared. As can be seen in Figure 1 , the IgG (serum dilution 1:80) of the $2 \mathrm{AD}$ cases presented bind to PK200 and to NF-H purified from either the PK or the axons of Torpedo cholinergic neurons. The $\operatorname{IgG}$ of one of the AD patients bind to an additional axonal and $\mathrm{PK}$ band whose size $(150 \mathrm{kDa})$ is similar to that of the medium molecular weight neurofilament protein (Fig. 1). The extent to which the levels of anti-Torpedo cholinergic NF-H IgG and anti-PK200 IgG in $A D$ sera correlate was investigated by quantitative immunoblot experiments in which the areas under the corresponding immunoblot peaks of the sera of $15 \mathrm{AD}$ patients were measured and compared. As shown in Figure 2, the levels of AD IgG to PK200 and to the NF-H of Torpedo cholinergic axons varied from individual to individual. However, the levels of these IgG in each AD patient were highly correlated $(R=0.92, p<0.001)$. A similar correlation was obtained between the individual AD IgG to PK200 and to cholinergic PK NF-H $(R=0.90, p<$ 0.001 ) and between the AD IgG that recognize axonal and $\mathrm{PK}$ NF-H $(R=0.90, p<0.001)$. Furthermore, the titers of the antiTorpedo cholinergic NF-H and anti-PK200 in each AD patient were similar. Most were detectable at serum dilutions higher than 1:160 (not shown).

To cvaluate the specificity of the anti-Torpedo cholinergic NF-H antibodies to AD, we performed the immunoblot assay 


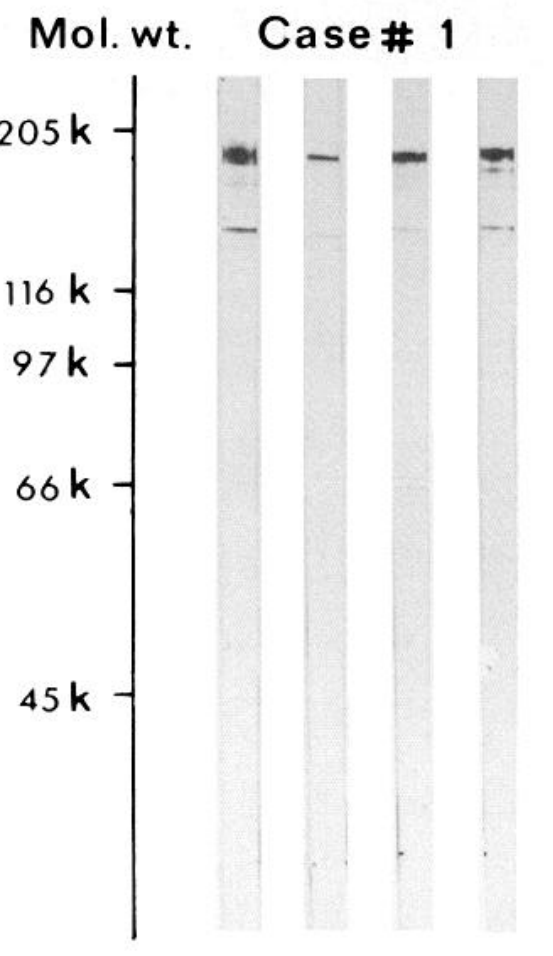

Antigen: Tot. NF Tot. NF
PK
Case\# 2

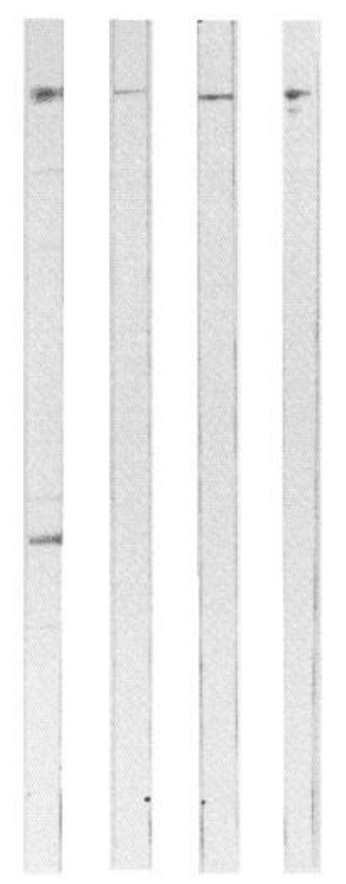

$\begin{array}{cc}\text { Tot. NF } & \text { Tot. NF } \\ \text { PK } & \text { AX }\end{array}$
Protein staining
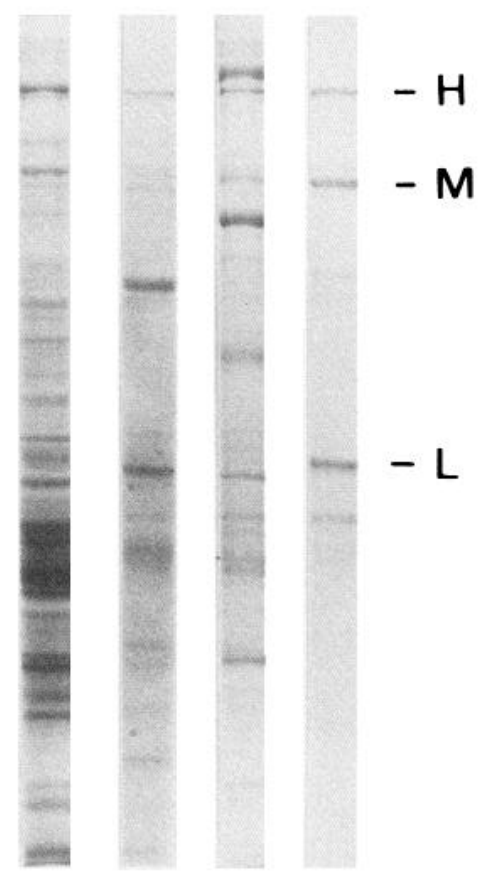

$\underset{\text { PK }}{\text { Tot. NF }} \stackrel{\text { Tot. NF }}{\text { AX }}$

Figure 1. Immunoblot assays of IgG directed against Torpedo cholinergic neurons and neurofilaments purified from these neurons in sera of 2 patients with AD. Sera (dilution 1:80) were immunoreacted with blots of electrophoresed PK (Tot. PK) and axons (Tot. AX) of Torpedo cholinergic neurons and with isolated neurofilaments which were purified from their perikarya $(N F P K)$ and axons $(N F A X)$. For experimental detail see Materials and Methods. The 4 lanes on the right correspond to Coomassie blue staining following polyacrylamide gel electrophoresis of the Torpedo cholinergic cell bodies and axons and their purified neurofilaments. The position of the heavy, medium, and light neurofilament subunits is indicated by $H, M$, and $L$. The molecular weight scale was generated by determining the position of marker proteins of known molecular weight.

with a large number of $\mathrm{AD}$ and normal control sera and with the sera of patients with other dementias, i.e., multi-infarct dementia and Parkinson's disease with dementia. Figure 3 represents the individual levels of IgG to NF-H in axons of electromotor neurons in sera (dilution 1:80) of $15 \mathrm{AD}$ patients, 15 normal age-matched controls, 11 multi-infarct dementia patients, and 8 Parksinson's disease patients with dementia. As can be seen, the median of the anti-NF-H IgG of the AD patients is 0.48 (arbitrary units), whereas those of the normal controls, the patients with Parkinson's disease with dementia, and the multi-infarct dementia patients are, respectively, $0.07,0.03$, and 0.0 . The observed difference between the AD patients and the other groups is statistically significant $(p<0.01$, Wilcoxon rank order test). Comparison of the average anti-NF-H IgG levels in these groups revealed that the AD sera contained higher antibody levels $(0.8 \pm 0.26$, average \pm SEM $)$ than did those of normal controls $(0.17 \pm 0.06)$ and those of patients with either multi-infarct dementia $(0.06 \pm 0.02)$ or Parkinson's disease with dementia $(0.22 \pm 0.14)$. It should, however, be noted that 2 of the 15 normal controls and 2 of the 9 patients with Parkinson's disease with dementia who were tested had antibody levels considerably higher than the respective averages of their groups (Fig. 3).

The extent to which the NF-H epitopes that are recognized by $\mathrm{AD} \mathrm{IgG}$ are specific to Torpedo cholinergic neurons was examined. To this end, neurofilaments were isolated from Tor- pedo spinal cord and from rat brain stem. These neurofilaments and those purified from Torpedo cholinergic neurons were immunoreacted with sera of $\mathrm{AD}$ patients and controls. Figure 4 depicts the immunoblots thus obtained with the sera of one $\mathrm{AD}$ patient and one normal control (dilution 1:80). As can be seen, the AD IgG reacted more strongly with Torpedo cholinergic NF-H than with Torpedo spinal cord and rat NF-H, whereas the control reacted weakly with rat NF-H and not at all with either of the Torpedo NF-H proteins. These findings were extended and quantitated by measurements of the levels of the anti-NF-H IgG in sera of $15 \mathrm{AD}$ patients and 15 controls. As shown in Figure 5, the anti-rat brain stem NF-H IgG levels of the $\mathrm{AD}$ patients and the normal controls were similar, as were their antibody levels to Torpedo spinal cord NF-H. The medians of the anti-rat NF-H IgG of the AD patients and the normal controls (respectively 0.25 and 0.16 ) and those of the anti-Torpedo spinal cord NF-H antibodies of these cases (respectively 0.32 and 0.17$)$ did not differ significantly $(p>0.1)$. Similar results were obtained utilizing AD and control sera at a dilution of $1: 40$ (not shown).

The finding that $\mathrm{IgG}$ of $\mathrm{AD}$ patients and controls bind similarly to NF-H from chemically heterogenous neurons and that the levels of IgG to NF-H of Torpedo cholinergic neurons are specifically high in $\mathrm{AD}$ patients suggests that $\mathrm{AD}$ sera contain a repertoire of anti-NF-H IgG and that only a subpopulation of these antibodies is specific to AD. This possibility was ex- 


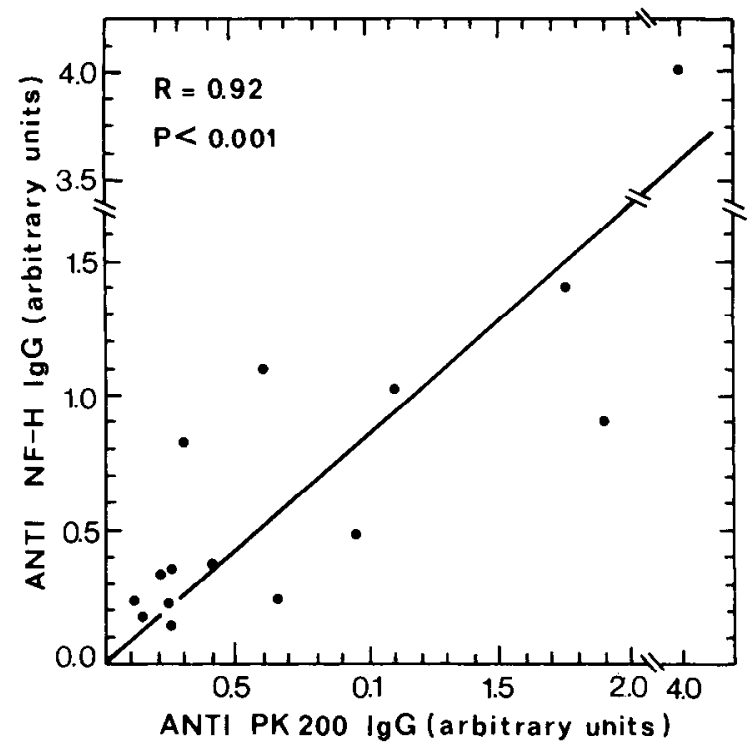

Figure 2. Correlation between the individual levels of IgG in AD sera directed against the PK200 antigen and the heavy neurofilament protein (NF-H) of Torpedo cholinergic neurons. Sera (dilution 1:80) from 15 AD patients were immunoreacted with blots of electrophoresed Torpedo cholinergic PK and purified neurofilaments which were isolated from the axons of these neurons. Results presented are the area (in arbitrary units) of the anti-PK200 and anti-NF-H immunoblot peaks of the individual cases as measured by a computerized densitometer.

amined by a normalization procedure in which the relative enrichment of IgG specific to Torpedo cholinergic NF-H in given sera was estimated from the ratio of anti-Torpedo cholinergic NF-H IgG to anti-Torpedo spinal cord NF-H IgG. As shown in Figure 6, the ratios of the anti-Torpedo cholinergic NF-H IgG to anti-Torpedo spinal cord NF-H IgG were found to be greater than 1 for 12 of the 15 AD cases examined, whereas only 1 of the 15 normal controls tested had such a ratio. This difference was statistically significant $(p<0.001)$. Three of the normal controls had nondetectable IgG against either the Torpedo cholinergic or the Torpedo spinal cord NF-H proteins, and their anti-NF-H $\lg (\mathrm{i}$ ratios were taken as $\mathrm{l}$ (see open symbols in Fig. 6). Similar results were obtained utilizing the ratio of anti-Torpedo cholinergic NF-H antibodies to anti-rat NF-H IgG in sera of AD patients and normal controls (not shown).

The antigenic properties of neurofilaments are affected by their degree of phosphorylation (Sternberger and Sternberger, 1983; Lee et al., 1987; Dahl et al., 1988). We therefore examined the possibility that dephosphorylation affects the binding of $A D$ IgG to Torpedo cholinergic NF-H. This was performed by comparing the binding of IgG from $14 \mathrm{AD}$ patients and 14 normal controls to phosphorylated and dephosphorylated Torpedo cholinergic NF-H. Figure 7 depicts the individual ratios of the levels of anti-dephosphorylated Torpedo cholinergic NF-H IgG (serum dilution 1:80) to anti-Torpedo cholinergic NF-H IgG for each case. As can be seen, the effect of dephosphorylation varied from individual to individual both in magnitude and direction. Dephosphorylation decreased the binding of $\operatorname{IgG}$ from 10 of the AD patients and increased the binding of $\operatorname{IgG}$ from 3; binding of IgG from one was unchanged. Of the 7 normal controls that had detectable anti-Torpedo cholinergic NF-H, the immunoreactivity of 2 was increased by dephosphorylation and that of 5 was decreased. The medians of the individual ratios of anti-

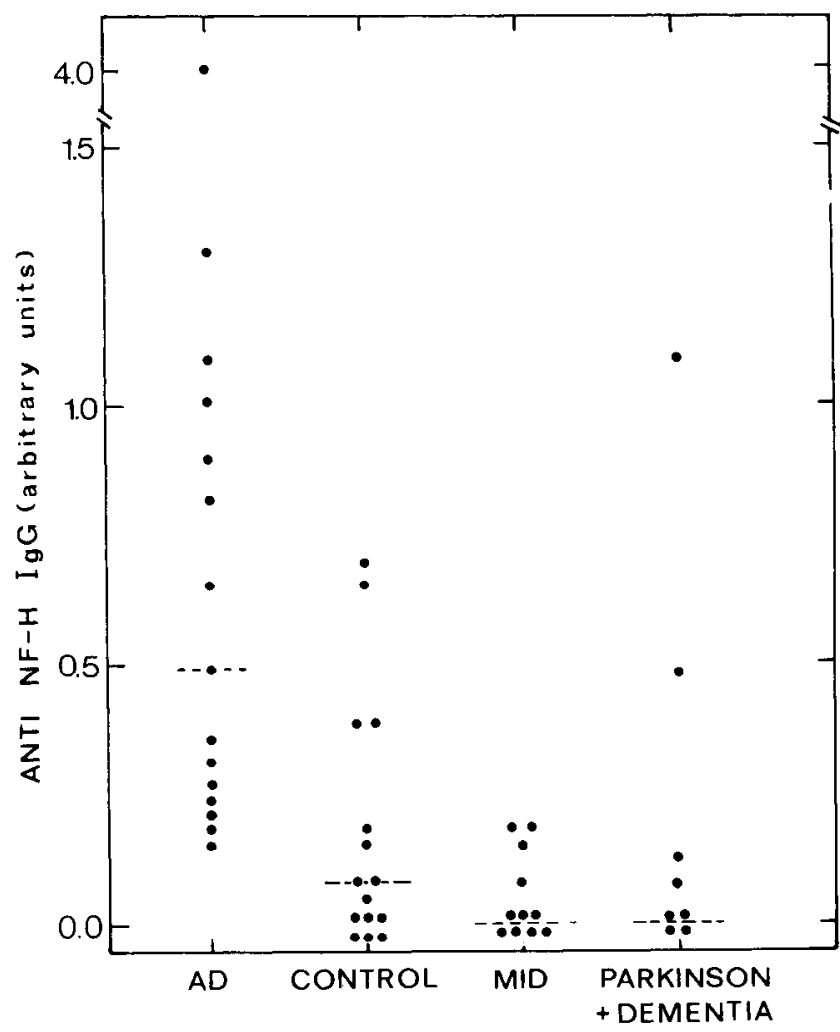

Figure 3. Individual levels of anti-Torpedo cholinergic NF-H IgG in sera of patients with dementia and normal controls. Sera (dilution 1:80) from patients with $\mathrm{AD}(n=15)$, multi-infarct dementia (MID; $n=11)$, Parkinson's disease with dementia $(n=8)$, and normal controls $(n=$ 15) were immunoreacted with blots of purified neurofilaments which were isolated from axons of Torpedo cholinergic neurons. The immunoblot assay was performed as described in Materials and Methods. Results presented are the area (in arbitrary units) of the anti-NF-H immunoblot peaks of the individual cases as measured by a computerized densitomter. Broken horizontal lines indicate medians.

dephosphorylated Torpedo cholinergic NF-H IgG to anti-Torpedo cholinergic NF-H were, respectively, 0.85 and 1.0 for the $\mathrm{AD}$ patients and the controls; the averages were, respectively, $0.85 \pm 0.15$ and $1.1 \pm 0.2$

\section{Discussion}

Our findings indicate that $\mathrm{IgG}$ from sera of AD patients bind to epitopes on the NF-H protein that are specific to Torpedo cholinergic neurofilaments. These IgG were detected far more frequently in $\mathrm{AD}$ patients than in healthy controls or patients with other common causes of dementia.

Examination of the protein composition of Torpedo neurofilaments revealed that they contain a triplet of polypeptides whose molecular weights $(200,150$, and $63 \mathrm{kDa})$ are similar to those of the corresponding mammalian neurofilament proteins Figs. 1, 4). Neurofilaments from Torpedo cholinergic axons and Torpedo spinal cord contain an additional protein whose molecular weight $(58 \mathrm{kDa})$ is similar to that of a protein associated with mammalian peripheral neurons (Zackroff, 1986). The neurofilament triplet proteins of Torpedo cholinergic PK were found to be associated with a number of additional proteins that have not yet been identified (Fig. 1). This protein pattern is similar to that of rat brain neurofilaments except that in Torpedo PK the molecular weight of the most prominent associated protein 
Figure 4. Immunoblot assays of IgG in an AD patient and a normal control pedo cholinergic neurons (TC), Torpedo spinal cord $(T S)$ and rat brain $(R B)$. Sera (dilution 1:80) were immunoreacted with blots of the indicated neurofilament preparations as described in Materials and Methods. The 3 lanes on the right correspond to Coomassie blue staining of electrophoresed neurofilaments whose heavy $(H)$, medium $(M)$, and light $(L)$ subunits are marked. The molecular weight scale was generated by determining the position of marker proteins of known molecular weight. directed against neurofilaments of Tor-

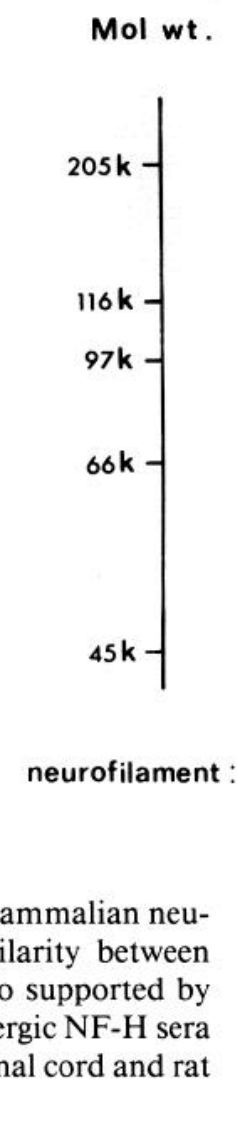

$(100 \mathrm{kDa})$ is larger than those associated with mammalian neurofilaments (Zackroff, 1986). The general similarity between Torpedo and mammalian neurofilaments is also supported by our recent observation that anti-Torpedo cholinergic NF-H sera prepared in rabbits cross-react with Torpedo spinal cord and rat brain NF-H (not shown).

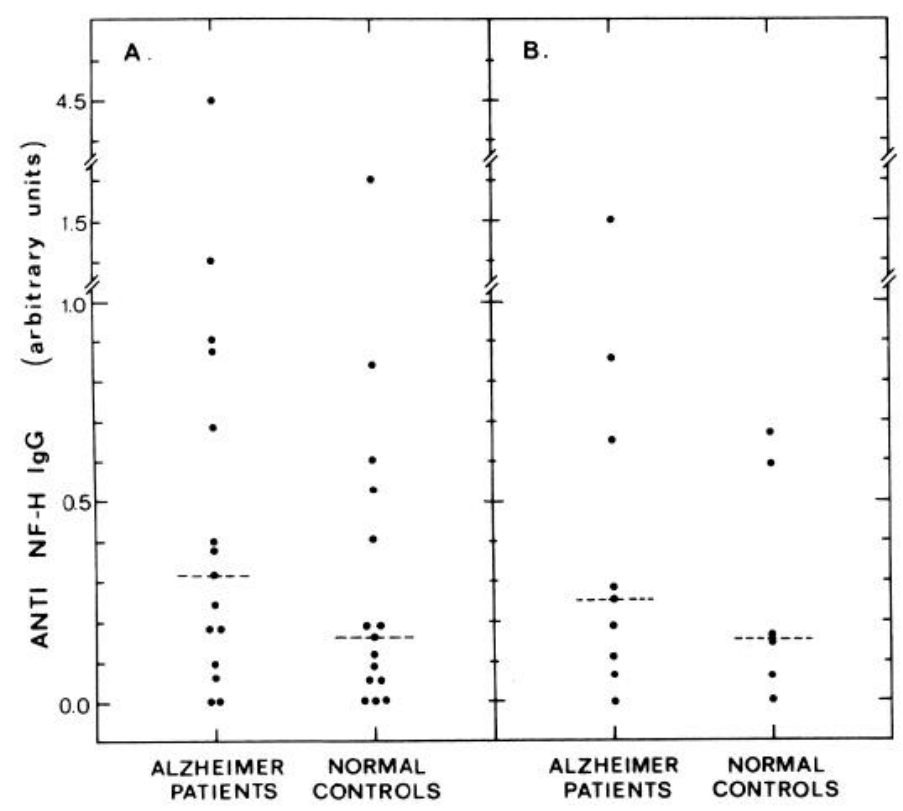

Figure 5. Individual levels of anti-Torpedo spinal cord $(A)$ and antirat brain NF-H IgG $(B)$ in sera of AD patients and normal controls. Sera (dilution 1:80) from AD patients and normal controls were immunoreacted with blots of electrophoresed neurofilaments which were isolated from Torpedo spinal cord and rat brain stem. The experiments were performed as described in Materials and Methods. Results presented are the areas (in arbitrary units) of the anti-Torpedo spinal cord and anti-rat brain NF-H immunoblot peaks of the individual cases as measured by a computerized densitometer. Broken horizontal lines indicate medians.
AD

Control

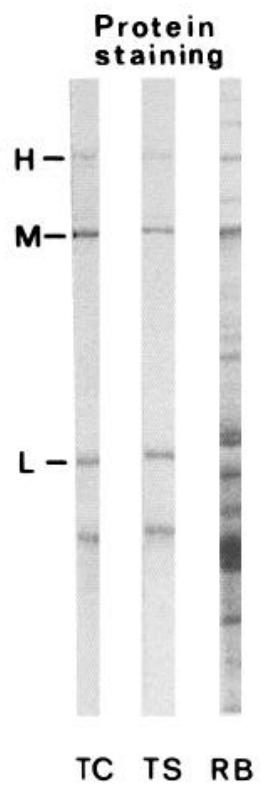

The findings that the levels of AD IgG directed against Torpedo cholinergic NF-H are significantly higher than those of normal and neurological controls (Fig. 3) and that, in contrast, these $\mathrm{IgG}$ bind similarly to NF-H isolated from Torpedo spinal cord and rat brain suggest that Torpedo cholinergic NF-H contain specific epitopes that are less abundant in the NF-H protein of chemically heterogenous neurons. About half of the AD cases examined also have IgG that recognize the 150 -kDa neurofilament subunit. It is therefore possible that this NF protein shares some of the cholinergic NF-H epitopes that are recognized by the $\mathrm{AD}$ IgG. The present findings also suggest that the cholinergic and the chemically heterogenous NF-H proteins have common domains that are recognized similarly by the AD and the control IgG. This is in accord with previous findings that $\mathrm{AD}$ and control IgG bind similarly to mammalian brain NF-H (Sotelo et al., 1980; Gajdusek, 1985; Stefansson et al., 1985; Karcher et al., 1986).

The individual levels of AD IgG directed against the NF-H protein of either the PK or the axons of Torpedo cholinergic neurons are highly correlated $(R=0.9, p<0.001)$, whereas $\mathrm{AD}$ $\mathrm{IgG}$ do not bind specifically to synaptosomes prepared from these neurons (Chapman et al., 1986, 1988). This suggests that the specific NF-H epitopes recognized by AD IgG reside in the cell bodies and axons but not in the nerve terminals of Torpedo cholinergic neurons. A high degree of correlation was found between the levels of AD IgG directed against Torpedo cholinergic NF-H obtained from the PK and axons of these neurons and anti-PK200 AD IgG (respectively $R=0.92, p<0.01$ and $R=0.98, p<0.01$ ). This suggests that PK200, which was initially identified as a Torpedo cholinergic antigen that is specifically recognized by AD IgG (Chapman et al., 1988), is the NF-H protein of these neurons.

It is not known whether AD patients also have antibodies that recognize other cytoskeletal proteins, particularly components of microtubules such as tubulin or the microtubule-associated protein Tau, which is a constituent of the paired helical filaments in AD (Grundke-Iqbal et al., 1986b; Goedert et al., 1988). Previous results suggest that AD IgG bind to a $55-\mathrm{kDa}$ protein in the cell bodies of Torpedo cholinergic neurons but 


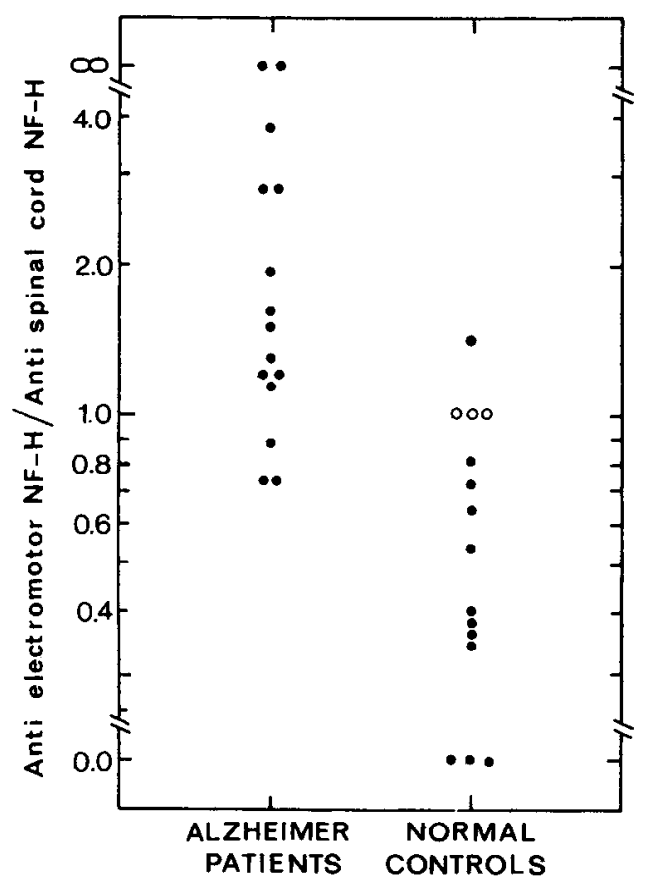

Figure 6. The ratio in AD patients $(n=15)$ and normal controls ( $n$ $=15$ ) of anti-Torpedo cholinergic neurons NF-H IgG to anti-Torpedo spinal cord NF-H IgG. Ratios presented were calculated utilizing the immunoblot data presented in Figures 3 and 5. For cases having no detectable IgG to either the cholinergic or the spinal cord NF-H, the ratios were assigned the values 0 and $\infty$, respectively. The ratio was taken as 1 for cases with no IgG against either protein (open symbols).

that similar IgG are present in normal control sera (Chapman et al., 1988). Furthermore, about one third of the AD patients and normal controls have $\operatorname{IgG}$ which bind to rat tubulin and to microtubular proteins with molecular weights between $50 \mathrm{kDa}$ and $55 \mathrm{kDa}$ (not shown). These observations suggest that some cases have antimicrotubular antibodies but that they are not specific to AD patients.

The diagnostic potential of the $\mathrm{AD}$ anti-Torpedo cholinergic $\mathrm{NF}-\mathrm{H}$ antibodies in differentiating $\Lambda \mathrm{D}$ patients from normal age-matched controls, from patients with other common causes of dementia (multi-infarct dementia and Parkinson's disease with dementia), and from patients with other relevant neurological disorders (cerebrovascular disease and Parkinson's disease) was examined. In order to account for the finding that IgG from $\mathrm{AD}$ patients and controls also recognize NF-H derived from chemically heterogenous neurons, the diagnostic potential of the specific anti-Torpedo cholinergic NF-H IgG was evaluated from the ratio of these IgG to IgG directed against NF-H from chemically heterogenous neurons. To minimize possible antigenic variation owing to species differences, the specificity of the anti-Torpedo cholinergic NF-H IgG to AD was evaluated from the ratios of these antibodies to those that recognize Torpedo spinal cord NF-H. This analysis revealed that $80 \%$ of the individual $\mathrm{AD}$ IgG bind more to the cholinergic NF-H protein than to Torpedo spinal cord NF-H as opposed to only about $10 \%$ of the normal controls and patients with Parkinson's disease with dementia and none of the other neurological cases (Fig. 6, Table 1). Similar results were obtained when the differences between anti-cholinergic NF-H and anti-spinal cord NF-H IgG levels in sera of AD patients and controls were compared (not shown). The proportion of AD patients with more anti-

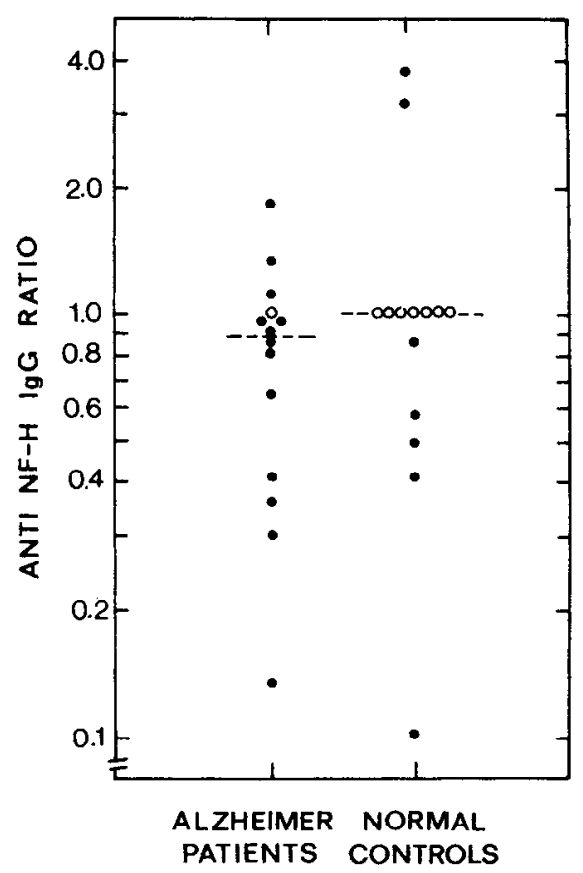

Figure 7. The cffcct of dephosphorylation on the binding of $A D$ and normal control IgG to Torpedo cholinergic NF-H. Results presented are the individual ratios of antidephosphorylated Torpedo cholinergic NF-H IgG to anti-Torpedo cholinergic NF-H IgG in sera (dilution 1:80) of 14 AD patients and 14 normal controls. The immunoblot experiments were performed as described in Materials and Methods. Open symbols correspond to cases with no detectable IgG either prior to or following dephosphorylation.

cholinergic NF-H IgG is close to the theoretical limit, since present clinical diagnostic procedures are only accurate to within $80 \%$ (McKhann et al., 1984). The approximately $10 \%$ of the normal controls and patients with Parkinson's disease with dementia who have anti-NF-H IgG ratios greater than 1 may represent presymptomatic $\mathrm{AD}$ cases or may alternatively be due to the presence of IgG not related to AD. These possibilities are presently bcing cxamincd by a prospective follow-up of these cases.

The nature of the Torpedo NF-H epitopes that are recognized by $A D \operatorname{IgG}$ is not known. Previous reports suggest that antigenically distinct variants of neurofilament proteins exist in different neurons (Kahn et al., 1987; Vitadello et al., 1987) and that some of the NF-H antigenic differences are due to variation in the extent of phosphorylation of this protein (Sternberger and Sternberger, 1983; Lee et al., 1987; Dahl et al., 1988). The present finding that exposure of Torpedo cholinergic NF-H to alkaline phosphatase has varying and opposing effects on the binding of individual AD IgG to Torpedo cholinergic NF-H (Fig. 7) suggests that some of the epitopes that are recognized by the $\mathrm{AD} \mathrm{IgG}$ are phosphorylated and some are dephosphorylated. AD patients have a repertoire of anti-Torpedo cholinergic NF-H IgG of which only a subpopulation is specific to $\mathrm{AD}$ (Fig. 6). It is not yet known which of the phosphorylated and dephosphorylated epitopes is recognized by the AD-specific IgG. Further studies that entail isolating the domains on Torpedo cholinergic NF-H to which the AD-specific IgG bind will enable direct examination of this question.

Recent immunohistochemical studies suggest that IgG obtained from sera and cerebrospinal fluid of $A D$ patients bind 
Table 1. Anti-Torpedo neurofilament IgG

\begin{tabular}{|c|c|c|c|c|c|c|}
\hline Sera & $n$ & $\begin{array}{l}\text { Anti-T. } \\
\text { cholinergic } \\
\text { NF-H } \\
\text { (arbitrary } \\
\text { units) }\end{array}$ & $\begin{array}{l}\text { Anti- } T \text {. } \\
\text { spinal cord } \\
\text { NF-H } \\
\text { (arbitrary } \\
\text { units) }\end{array}$ & $\begin{array}{l}\text { Ratio } \\
\text { Anti-T. } \\
\text { cholinergic } \\
\text { NF-H/ } \\
\text { Anti-T. spinal } \\
\text { NF-H }\end{array}$ & $\begin{array}{l}\text { Ratio } \\
\text { Anti-T. } \\
\text { cholinergic } \\
\text { NF-H/ } \\
\text { Anti-T. } \\
\text { spinal } \\
\text { NF-H > } 1 \\
\text { Positive } \\
\text { cases (\%) }\end{array}$ & $p^{a}$ \\
\hline $\mathrm{AD}$ & 15 & $0.80 \pm 0.26$ & $0.70 \pm 0.31$ & $2.77 \pm 0.79$ & $12 / 15(80)$ & \\
\hline Normal control & 15 & $0.17 \pm 0.06^{h}$ & $0.32 \pm 0.11$ & $0.58 \pm 0.11^{b}$ & $1 / 15(7)$ & $<0.001$ \\
\hline Multi-infarct dementia & 11 & $0.06 \pm 0.02^{b}$ & $0.15 \pm 0.07^{d}$ & $0.70 \pm 0.13^{b}$ & $0 / 11(0)$ & $<0.001$ \\
\hline $\begin{array}{l}\text { Parkinson's disease } \\
\text { with dementia }\end{array}$ & 8 & $0.22 \pm 0.14^{c}$ & $0.43 \pm 0.33$ & $0.91 \pm 0.08^{c}$ & $1 / 8 \quad(12)$ & $<0.003$ \\
\hline $\begin{array}{l}\text { Cerebrovascular } \\
\text { disease }\end{array}$ & 7 & $0.24 \pm 0.20^{c}$ & $0.27 \pm 0.23$ & $0.93 \pm 0.05^{d}$ & $0 / 7 \quad(0)$ & $<0.001$ \\
\hline Parkinson's disease & 5 & $0.14 \pm 0.11^{c}$ & $0.22 \pm 0.14$ & $0.74 \pm 0.19^{c}$ & $0 / 5 \quad(0)$ & $<0.004$ \\
\hline
\end{tabular}

The levels of IgG to NF-H isolated from axons of Torpedo electromotor neurons and from Torpedo spinal cord werc measured by immunoblot assays as described in Materials and Methods. They are presented as mean peak area \pm SEM of the indicated number of cases. The results of the $\mathrm{AD}$ patients were compared to those of the other groups by means of a Wilcoxon rank order test. The ratio of anti-Torpedo cholinergic NF-H to anti-Torpedo spinal cord NF-H was calculated for each case. Results presented are the average \pm SEM for each group. For cases having no detectable IgG to either the cholinergic or the spinal cord $\mathrm{NF}-\mathrm{H}$, the ratios were assigned the values 0 and $\infty$, respectively. The ratio was taken as 1 for cases with no IgG against either protein.

"The $p$ values correspond to a comparison of the Alzheimer data to those of the other groups by means of an appropriate statistical test (Fisher's exact test or chi-square test).

${ }^{\prime \prime} p<0.001 ; " p<0.01 ;{ }^{d} p<0.05$.

specifically to cholinergic neurons in the basal forebrain of rats (Fillit et al., 1985; McRae-Degueurce et al., 1987). Although the antigens with which these antibodies interact have not yet been identified, it is tempting to suggest that mammalian cholinergic neurons contain epitopes similar to those of Torpedo cholinergic $\mathrm{NF}-\mathrm{H}$ and that at least part of the immunohistochemically detected anticholinergic antibodies are directed against mammalian cholinergic NF-H. Preliminary experiments in which we examined this assertion by immunoblot assays that utilized homogenates of human hippocampus and cortex and $\mathrm{AD}$ and normal control sera (10 cases in each group at dilution $1: 40$ ) revealed the presence of $\operatorname{IgG}$ to $200-\mathrm{kDa}$ proteins in less than half of the AD cases examined and in a similar number of the normal controls (not shown). These findings are consistent with the results obtained utilizing purified rat neurofilaments as antigen (Fig. 5). Both observations suggest that utilization of either brain homogenates or purified mammalian neurofilaments is not sufficient for the detection of AD-specific IgG. To circumvent this difficulty we have begun to prepare monoclonal antibodies to the domains on Torpedo cholinergic NF-H that are recognized specifically by the $A D$ IgG.

The immunological trigger for the formation of anti-Torpedo cholinergic NF-H IgG and the extent to which these antibodies are involved in the development of cholinergic dysfunction in $\mathrm{AD}$ are not yet known. Interference with axonal transport of neurofilaments has been proposed as a common pathogenetic mechanism in $\mathrm{AD}$ and in certain other diseases of the CNS (Gajdusek, 1985). It is therefore tempting to suggest that the presently described AD IgG contribute to the cholinergic degeneration underlying this disease by specifically affecting axonal transport in cholinergic neurons. Future studies of the correlation between clinical, histological, and neurochemical parameters in AD and the levels of anticholinergic NF-H an- tibodies in the sera, cerebrospinal fluid, and brains of the patients may reveal whether these antibodies have a significant role in the disease.

\section{References}

Anderton, B. H., D. Breinburg, M. J. Downes, P. J. Green, B. E. Tomlinson, J. Ulrich, N. Woods and J. Kahn (1982) Monoclonal antibodies show that neurofibrillary tangles and neurofilaments share antigenic determinants. Nature 298: 84-86.

Blessed, G., B. E. Tomlinson, and M. Roth (1968) The association between quantitative measures of dementia and of senile change in the cerebral grey matter of elderly subjects. Br. J. Psychiatry 114 : 797-811.

Chapman, J., A. D. Korczyn, M. Ilareuveni, and D. M. Michaelson (1986) Antibodies to cholinergic cell bodies in Alzheimer's disease. In Alzheimer's and Parkinson's Diseases, A. Fisher, I. Hanin, and C. Lachman, eds., pp. 329-336, Plenum, New York.

Chapman, J., O. Bachar, A. D. Korczyn, E. Wertman, and D. M. Michaelson (1988) Antibodies to cholinergic neurons in Alzheimer's disease. J. Neurochem. 51: 479-485.

Colton, T. (1974) Statistics in Medicine. Little, Brown, Boston.

Coyle, J. T., D. L. Price, and M. R. DeLong (1983) Alzheimer's disease: A disorder of cortical cholinergic innervation. Science 219: 11841190.

Dahl, D., B. Labkovsky, and A. Bigmani (1988) Neurofilament phosphorylation in axons and perikarya: Immunofluorescence study of the rat spinal cord and dorsal root ganglia with monoclonal antibodies. J. Comp. Neurol. 271: 445-450.

Dowdall, M. J., G. Fox, K. Wachtler, V. P. Whittaker, and H. Zimmermann (1976) Recent studies on the comparative biochemistry of the cholinergic neuron. Cold Spring Harbor Symposium on Quantitative Biology XL, 65-81.

Fillit, H., V. N. Luine, B. Reisberg, R. Amador, B. McEwen, and J. B. Zabriske (1985) Studies of the specificity of antibrain antibodies to Alzheimer's disease. In Senile Dementia of the Alzheimer Type, J. T. Hutton and A. D. Kenny, eds., pp. 307-318, Liss, New York.

Francis, P. T., A. M. Palmer, N. R. Sims, D. M. Bowen, A. M. Davison, M. M. Esiri, D. Neary, J. S. Snowden, and G. K. Wilcock (1985) 
Neurochemical studies of early-onset Alzheimer's disease: Possible influence on treatment. New Engl. J. Med. 313: 7-11.

Gajdusek, D. C. (1985) Hypothesis: Interference with axonal transport of neurofilament as a common pathogenetic mechanism in certain diseases of the central nervous system. New Engl. J. Med. 312: 714719.

Goedert, M., C. M. Wischik, R. A. Crowther, J. E. Waler, and A. Klug (1988) Cloning and sequencing of the cDNA encoding a core protein of the paired helical filaments of Alzheimer's disease: Identification as the microtubule-associated Protein Tau. Proc. Natl. Acad. Sci. USA 85: 4051-4055.

Grundke-Iqbal, I., K. Iqbal, Y. C. Tung, M. Quinlan, H. M. Wisniewski, and L. I. Binder (1986a) Abnormal phosphorylation of the microtubule-associated protein (tau) in Alzheimer cytoskeletal pathology. Proc. Natl. Acad. Sci. USA 83: 4913-4917.

Grundke-Iqbal, I., K. Iqbal, M. Quinlan, Y.-C. Tung, M. S. Zaidi, and II. M. Wisniewski (1986b) Microtubule-associated Protein Tau-a component of Alzheimer paired helical filaments. J. Biol. Chem. 261: 6084-6089.

Kahn, J., B. H. Anderton, C. C. J. Miller, J. N. Wood, and M. M. Esiri (1987) Staining with monoclonal antibodies to neurofilaments distinguishes between subpopulations of neurofibrillary tangles, between groups of axons and between groups of dendrites. J. Neurol. 234:241246.

Karcher, D., B. S. Soler Federsspiel, F. D. Lowenthal, F. Franck, and A. Lowenthal (1986) Anti-neurofilament antibodies in blood of patients with neurological diseases. Acta Neuropath. (Berl.) 72: 82-85.

Kushner, P. D. (1984) A library of monoclonal antibodies to Torpedo cholinergic synaptosomes. J. Neurochem. 43: 775-786.

Laemmli, U.K. (1970) Cleavage of structural proteins during assembly of the head of bacteriophage T4. Nature 227: 680-685.

Lee, V. M. Y., M. J. Carden, W. W. Schlaepfer, and J. Q. Trojanowski (1987) Monoclonal antibodies distinguish several differentially phosphorylated states of the two largest rat neurofilament subunits (NF-H and NF-M) and demonstrate their existence in the normal nervous system of adult rats. J. Neurosci. 7: 3474-3488.

Lowry, O. H., N. J. Rosebrough, A. L. Farr, and R. J. Randall (1951) Protein measurement with the Folin phenol reagent. J. Biol. Chem. 193: 265-275.

Markwell, M. A. K., S. M. Haas, L. C. Neiber, and N. E. Talbert (1978) A modification of the Lowry procedure to simplify protein determination in membrane and lipoprotein samples. Anal. Biochem. 87: 206-210.

McKhann, G., D. Drachmann, M. Folstein, R. Katzman, D. Price, and E. M. Stadlan (1984) Clinical diagnosis of Alzheimer's disease. Neurology 34: 939-944.

McRae-Degueurce, A., S. Booj, N. K. Haglid, L. Rosengren, J. E. Karlsson, I. Karlsson, A. Wallin, L. Svennerholm, C. G. Gottfries, and A. Dahlstrom (1987) Antibodies in cerebrospinal fluid of some Alzheimer disease patients recognize cholinergic neurons in the rat cental nervous system. Proc. Natl. Acad. Sci. USA 84: 9214-9218.

Nandy, K. (1978) Brain-reactive antibodies in aging and senile dementia. In Alzheimer's Disease. Senile Dementia and Related Dis- orders, Aging, Vol. 7, R. Katzman, R. D. Terry and K. L. Bick, eds. pp. 503-511, Raven, New York.

Patrick, J., and J. Lindstrom (1973) Autoimmune response to acetylcholine receptors. Science 180: 871-879.

Perry, G., R. Friedman, D. H. Kang, V. Manetto, L. Autilio-Gambetti, and P. Gambetti (1987) Antibodies to the neuronal cytoskeleton are elicited by Alzheimer paired helical filament fractions. Brain Res. 420: 233-242.

Schlaepfer, W. W., and L. A. Freeman (1978) Polypeptides of neurofilaments isolated from rat peripheral and central nervous systems. J. Cell Biol. 78: 653-662.

Selkoe, D. J., and C. R. Abraham (1986) Isolation of paired helical filaments and amyloid fibers from human brain. Methods Enzymol. 134: $388-404$.

Shelanski, M. L., F Gaskin, and C. R. Cantor (1973) Microtubule assembly in the absence of added nucleotides. Proc. Natl. Acad. Sci. USA 70: 765-768.

Sims, N. R., D. M. Bowen, S. J. Allen, C. C. T. Smith, D. Neary, D. J. Thomas, and A. N. Davison (1983) Presynaptic cholinergic dysfunction in patients with dementia. J. Neurochem. 40:503-509.

Sotelo, J., C. J. Gibbs, and D. C. Gajdusek (1980) Autoantibodies against axonal neurofilaments in patients with Kuru and CreutzfeldtJakob disease. Science 210: 190-193.

Stefansson, K., L. S. Marton, M. E. Dieperink, G. K. Molnar, W. W. Schlaepfer, and C. M. Helgason (1985) Circulating autoantibodies to the 200,000-dalton protein of neurofilaments in the serum of healthy individuals. Science 228: 1117-1119.

Sternberger, L. A., and N. II. Sternberger (1983) Monoclonal antibodies distinguish phosphorylated and nonphosphorylated forms of neurofilaments in situ. Proc. Natl. Acad. Sci. USA 80: 6126-6130.

Sternberger, N. H., L. A. Sternberger, and J. Ulrich (1985) Aberrant neurofilament phosphorylation in Alzheimer disease. Proc. Natl. Acad. Sci. USA 82: 4274-4276.

Towbin, H., T. Staehlin, and J. Gordon (1979) Electrophoretic transfer of proteins from polyacrylamide gels to nitrocellulose sheets: Procedures and some applications. Proc. Natl. Acad. Sci. USA 76: 4350 4354.

Vitadello, M., C. Triban, M. Fabris, M. Dona, A. Gorio, and S. Schiaffino (1987) A developmentally regulated isoform of 150,000 molecular weight neurofilament protein specificially expressed in autonomic and small sensory neurons. Neuroscience 23: 931-941.

Watts, H., P. G. E. Kennedy, and M. Thomas (1981) The significance of anti-neuronal antibodies in Alzheimer's disease. J. Neuroimmunol. 1: $107-116$.

Willard, M., and C. Simon (1981) Antibody decoration of neurofilaments. J. Cell Biol. 89: 198-205.

Zackroff, R. V. (1986) Isolation of intermediate filaments from neuronal tissues by cycles of assembly/disassembly. Methods Enzymol. 134: 371-380.

Zemcov, A., L. L. Barclay, D. Brush, and J. P. Blass (1984) Computerized data base for evaluation and follow-up of demented outpatients. J. Am. Geriatric Soc. 32: 801-823. 